\title{
Dynamic Control of Internal Force for Visco-Elastic Contact Grasps
}

\section{Prattichizzo}

\author{
Dip. Ing. Informazione \\ Università di Siena \\ Siena, Italy 53100 \\ prattichizzo@ing.unisi.it
}

P. Mercorelli

Dip. Ing. Informazione

Università di Siena

Siena, Italy 53100

mercorel@ing.unisi.it
F. Barbagli

PERCRO

Scuola Superiore S. Anna

Pisa, Italy 56100

fed@sssup.it

\begin{abstract}
This paper deals with robotic grasps exhibiting viscoelastic contact interactions with the manipulated object. Such interactions are frequent in advanced robotic applications since in many cases, i.e. when a great precision is needed, elasticity cannot be neglected. This is particularly true in medical applications, as in tele-surgery or in laparoscopy, where precision is obviously basic.

The control of internal forces for visco-elastic grasps is explored in this work. This is an important issue in tele-operated robotics (tele-surgery and so forth). The presence of non-negligible compliance at the contacts, implies that the object dynamics cannot be neglected when attempting to control internal forces without affecting the object position.

A geometric approach to derive a control law dynamically decoupling the internal force control action from the object dynamics is proposed.
\end{abstract}

\section{Introduction}

This paper deals with robotic grasps exhibiting viscoelastic contact interactions with the manipulated object. Such interactions are frequent in advanced robotic applications since in many cases, i.e. when a lot of precision is needed, elasticity cannot be neglected.

This is particularly true in medical applications, as in tele-surgery or in laparoscopy, where precision is obviously basic. In such applications in fact the aim is to combine the capabilities of the surgeon and those of a robot, such as precision, to obtain a better overall result $[6,5]$.

A possible task in tele-surgery systems is to squeeze parts of tissue of the patient's organ to be operated in order to exert a cutting action, see fig.1. Such squeezing action often interacts with the tissue's position because

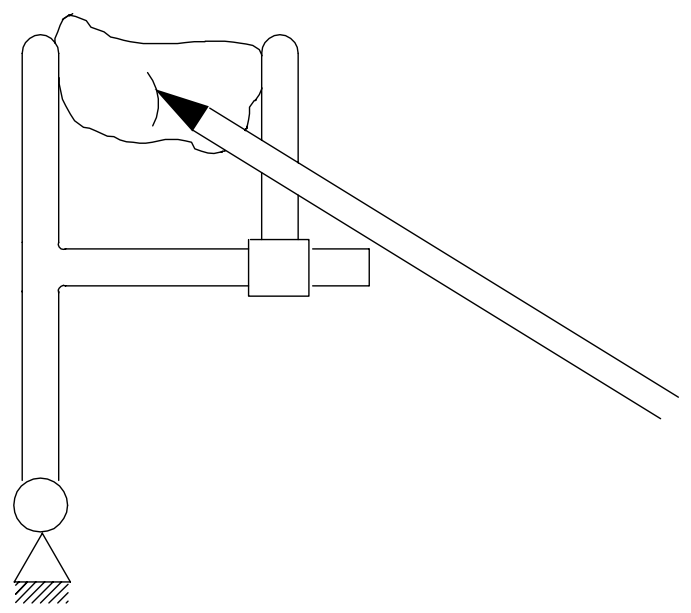

Figure 1: Micromanipulation of internal tissues in surgery or laparoscopy.

of the internal forces for visco-elastic grasps. The interaction can result in an unacceptable lack of precision since the tissue undergoing surgery should be still for obvious safety requirements [12].

Note that in industry, visco-elastic contacts occur whenever there is a need of assembling and manipulating non-rigid (rubber or plastic) parts. Moreover modeling contact elasticity is mandatory not only for deformable manipulated parts but also for soft fingertips in robotics hands as those of Akella and Cutkoski, [1].

This work is focused on the control of internal forces for contact-compliant grasps with general kinematics $[10,13,7]$. Internal forces play a key role in controlling the grasp. In force-closure grasps, a convenient control of internal forces guarantees that the whole vector of contact forces complies with contact friction constraints notwithstanding disturbances acting on the manipulated object. 


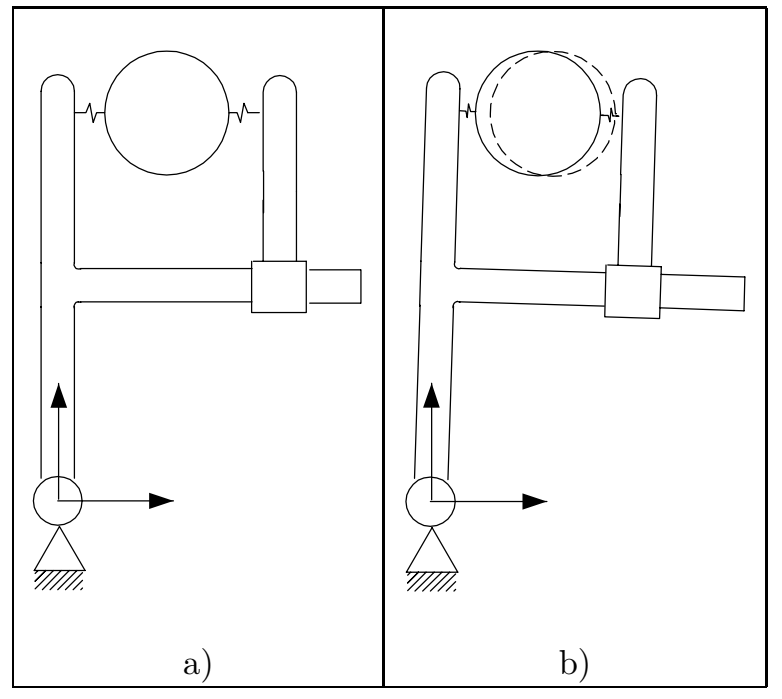

Figure 2: Deformable contacts: squeezing action of the prismatic joint.

If the manipulation system can be modeled by rigidbody contacts, usually the object dynamics is intentionally ignored to design the internal-force controller. On the other hand, for the aforementioned examples of advanced manipulation tasks, the object dynamics cannot be disregarded at all. As an example consider the manipulation system of fig. 1 and model the complaint contacts with visco-elastic lumped parameters as depicted in fig.2-a). A control policy ignoring object dynamics, for instance a force step on the prismatic joint, squeezes the manipulated object but an undesired and dangerous transient motion of the object arises.

The aim of this work is to fix such a problem. A feedback controller which perfectly decouples the internal force control loop from the object dynamics is proposed.

\section{Background}

This paper is based on the linearized dynamics of grasps with general kinematics derived in $[10,11]$ where system-theoretic structural properties were investigated. Some of these results along with notation are here summarized for the reader's convenience.

Let $\mathbf{q} \in \mathbb{R}^{q}$ be the vector of joint positions, $\tau \in$ $\mathbb{R}^{q}$ the vector of joint generalized forces, $\mathbf{u} \in \mathbb{R}^{d}$ the vector locally describing the position and the orientation of a frame attached to the object and finally $\mathbf{w} \in \mathbb{R}^{d}$ the vector of forces and torques resultant from external forces acting on the object.

Let further introduce the vector $\mathbf{t} \in \mathbb{R}^{t}$ whose components include contact forces and torques. We assume that contact forces arise from a lumped-parameter model of visco-elastic phenomena at the contacts, summarized by a stiffness matrix $\mathbf{K}$ and a damping matrix B. Notice that the lumped parameter model of contact interactions makes the identification procedure feasible with a reasonable amount of computations.

The Jacobian $\mathbf{J}$ and the grasp matrix $\mathbf{G}$ are defined as the linear maps relating the velocities of the contact points on the links and on the object, to the joint and object velocities, respectively, cf. [4].

It should be remarked that, according to [10], by general manipulation or grasp systems we mean mechanical devices with any $\mathbf{J}$ and $\mathbf{G}$ such that $\operatorname{ker}\left(\mathbf{G}^{T}\right) \neq \mathbf{0}$ (i.e. there does not exist any free motion of the object).

Consider a reference equilibrium configuration $(\mathbf{q}=$ $\mathbf{q}_{o}, \mathbf{u}=\mathbf{u}_{o}, \dot{\mathbf{q}}=\dot{\mathbf{u}}=\mathbf{0}, \tau=\tau_{o}$ and $\mathbf{t}=\mathbf{t}_{o}$ ), such that $\tau_{o}=\mathbf{J}^{T} \mathbf{t}_{o}$ and $\mathbf{w}_{o}=-\mathbf{G t}_{o}$. The linear approximation of the manipulation system dynamics in the neighborhood of such an equilibrium can be written as

$$
\dot{\mathbf{x}}=\mathbf{A} \mathbf{x}+\mathbf{B}_{\tau} \tau^{\prime}+\mathbf{B}_{w} \mathbf{w}^{\prime}
$$

where state and input vectors are defined as the departures from the reference equilibrium configuration: $\mathbf{x}=\left[\left(\mathbf{q}-\mathbf{q}_{o}\right)^{T}\left(\mathbf{u}-\mathbf{u}_{o}\right)^{T} \dot{\mathbf{q}}^{T} \dot{\mathbf{u}}^{T}\right]^{T}, \tau^{\prime}=\tau-\mathbf{J}^{T} \mathbf{t}_{o}$ $\mathbf{w}^{\prime}=\mathbf{w}+\mathbf{G t}_{o}$, and

$\mathbf{A}=\left[\begin{array}{cc}\mathbf{0} & \mathbf{I} \\ \mathbf{L}_{k} & \mathbf{L}_{b}\end{array}\right] ; \quad \mathbf{B}_{\tau}=\left[\begin{array}{c}\mathbf{0} \\ \mathbf{0} \\ \mathbf{M}_{h}^{-1} \\ \mathbf{0}\end{array}\right] ; \quad \mathbf{B}_{w}=\left[\begin{array}{c}\mathbf{0} \\ \mathbf{0} \\ \mathbf{0} \\ \mathbf{M}_{o}^{-1}\end{array}\right]$, being $\mathbf{M}_{h}$ and $\mathbf{M}_{o}$ the inertia matrices of the manipulator and the object, respectively. To simplify notation we will henceforth omit the prime in $\tau^{\prime}$ and $\mathbf{w}^{\prime}$.

Assuming that local variations of gravity forces on system dynamics, of the Jacobian and of the grasp matrices are negligible for small displacements $\delta \mathbf{u}, \delta \mathbf{q}$, simple expressions are obtained, cf. [10], for $\mathbf{L}_{k}=-\mathbf{M}^{-1} \mathbf{P}_{k}$ and $\mathbf{L}_{b}=-\mathbf{M}^{-1} \mathbf{P}_{b}$, where $\mathbf{M}=\operatorname{diag}\left(\mathbf{M}_{h}, \mathbf{M}_{o}\right), \mathbf{P}_{k}=$ $\mathbf{S}^{T} \mathbf{K S}, \mathbf{P}_{b}=\mathbf{S}^{T} \mathbf{B S}$, and $\mathbf{S}=\left[\mathbf{J}-\mathbf{G}^{T}\right]$.

\section{Controlled outputs: internal forces}

The control of contact forces $\mathbf{t}$ is a fundamental part of the manipulation control problem. Contact forces allows the manipulator to maintain the grasp, rejecting external disturbance $\mathbf{w}$ and controlling the object motion. In $[10,11]$ the reachable subspace of contact forces as outputs of the dynamic system (1) was studied.

Consider the departures of contact force vector $\mathbf{t}$ from the reference equilibrium $\mathbf{t}_{o}$, define $\mathbf{t}^{\prime}$ (henceforth t) as its first order approximation which in [10] was computed as an output of the linearized model (1): $\mathbf{t}=\mathbf{C}_{t} \mathbf{x}$ where $\mathbf{C}_{t}=\left[\mathbf{K J}-\mathbf{K G}^{T} \mathbf{B J}-\mathbf{B G}^{T}\right]$; and assume that stiffness matrix $\mathbf{K}$ and damping matrix $\mathbf{B}$ are proportional, which is customary in mechanical vibration analysis (see e.g. [8]). 
In [3] it was shown that in grasps with general kinematics not all the internal forces are controllable. Thus an analysis of their reachable set is needed in order to specify consistent control goals. In [11] the authors, starting from the linearized dynamics, define the reachable internal forces subspace $\mathcal{R}_{t i, \tau}$ as the intersection between the reachable subspace of all the contact force and the null space of the grasp matrix:

$$
\mathcal{R}_{t i, \tau}=\mathcal{R}_{t, \tau} \cap \operatorname{ker}(\mathbf{G}) .
$$

Moreover, in terms of column space, they showed that

$$
\begin{aligned}
& \mathcal{R}_{t i, \tau}=\operatorname{im}\left(\mathbf{P}_{N G} \mathbf{C}_{t}\right)=\operatorname{im}\left(\mathbf{P}_{N G} \mathbf{K J}\right) \\
& \text { where } \mathbf{P}_{N G}=\mathbf{I}-\mathbf{K G}^{T}\left(\mathbf{G K G}^{T}\right)^{-1} \mathbf{G} .
\end{aligned}
$$

According to this result, the subspace of reachable internal forces is obtained by the projector $\mathbf{P}_{N G}$ (on the null space of $\mathbf{G}$ ) acting on the column space of $\mathbf{C}_{t}$. By the way, observe that the previous formula states the equality of $\mathcal{R}_{t i, \tau}$ with the active forces in [3] and the asymptotically reachable forces in [11].

Finally, the regulated force output $\mathbf{e}_{t i}$ is chosen as the projection of the force vector $\mathbf{t}$ onto the null space of $\mathbf{G}$, i.e. the reachable internal contact forces:

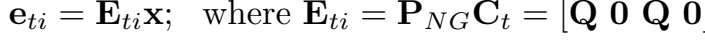

$$
\begin{aligned}
& \text { and } \mathbf{Q}=\left(\mathbf{I}-\mathbf{K G}^{T}\left(\mathbf{G} \mathbf{K G}^{T}\right)^{-1} \mathbf{G}\right) \mathbf{K J} \text {. }
\end{aligned}
$$

It might be worthwhile to emphasize that

$$
\mathcal{R}_{t i, \tau}=\operatorname{im}\left(\mathbf{E}_{t i}\right) .
$$

\section{Decoupled output: object motions}

Rigid-body kinematics are of particular interest in the control of manipulation systems. They do not involve visco-elastic deformations of bodies, thus they can be regarded as low-energy motions. In fact, rigid-body kinematics represent the easiest way to move the object.

Rigid-body kinematics have been studied in a quasistatic setting in [4] and in terms of unobservable subspaces from contact forces in [10]. In both cases rigid kinematics were described by a matrix $\boldsymbol{\Gamma}$ whose columns form a basis for $\operatorname{ker}\left[\mathbf{J}-\mathbf{G}^{T}\right]=\operatorname{im}(\boldsymbol{\Gamma})$ where

$$
\begin{aligned}
& \boldsymbol{\Gamma}=\left[\begin{array}{ccc}
\boldsymbol{\Gamma}_{r} & \boldsymbol{\Gamma}_{q c} & \mathbf{0} \\
\mathbf{0} & \boldsymbol{\Gamma}_{u c} & \boldsymbol{\Gamma}_{i}
\end{array}\right], \\
& \text { and } \mathbf{J} \boldsymbol{\Gamma}_{q c}=\mathbf{G}^{T} \boldsymbol{\Gamma}_{u c}
\end{aligned}
$$

being $\boldsymbol{\Gamma}_{r}$ a basis matrix (b.m.) of the subspace of redundant manipulator motions $\operatorname{ker}(\mathbf{J}), \boldsymbol{\Gamma}_{i}$ a b.m. of the subspace of indeterminate object motions $\operatorname{ker}\left(\mathbf{G}^{T}\right)$, and $\boldsymbol{\Gamma}_{q c}$ and $\boldsymbol{\Gamma}_{u c}$ conformal partitions of a complementary basis matrix ${ }^{1}$.

\footnotetext{
${ }^{1} \mathbf{W}$ is called a complementary basis matrix of $\mathcal{V}$ to $\mathcal{X}$ if it is f.c.r. and $\operatorname{im}(\mathbf{W}) \oplus \mathcal{V}=\mathcal{X}$.
}

The column space of $\boldsymbol{\Gamma}_{\mathbf{c}}=\left[\begin{array}{l}\boldsymbol{\Gamma}_{q c} \\ \boldsymbol{\Gamma}_{u c}\end{array}\right]$ consists of coordinated rigid-body motions of the mechanism, for the manipulator $\left(\boldsymbol{\Gamma}_{q c}\right)$ and the object $\left(\boldsymbol{\Gamma}_{u c}\right)$ components. Physically rigid-body displacements do not involve variation of contact forces.

In [10], it has been shown that rigid-body motions are reachable, i.e. they belong to the space of reachability of linear system (1) with input the vector of joint generalized forces $\tau$. Notice that the rigid-body subspace is only a subspace of the reproducible one which also contains motions due to deformations of elastic elements in the model.

The object-position regulated output $\mathbf{e}_{u c}$ is chosen as the projection, through $\boldsymbol{\Gamma}_{u c}^{P}$, of object positions $\mathbf{u}$ onto the subspace of rigid-body object motions $\operatorname{im}\left(\boldsymbol{\Gamma}_{u c}\right)$ :

$$
\begin{aligned}
& \mathbf{e}_{u c}=\mathbf{E}_{u c} \mathbf{x} ; \quad \text { where } \mathbf{E}_{u c}=\boldsymbol{\Gamma}_{u c}^{P}\left[\begin{array}{llll}
\mathbf{0} & \mathbf{I} & \mathbf{0} & \mathbf{0}
\end{array}\right] \\
& \text { and } \boldsymbol{\Gamma}_{u c}^{P}=\boldsymbol{\Gamma}_{u c}\left(\boldsymbol{\Gamma}_{u c}^{T} \boldsymbol{\Gamma}_{u c}\right)^{-1} \boldsymbol{\Gamma}_{u c}^{T} .
\end{aligned}
$$

\section{An example of internal force control}

The following example shows the behaviour of an internal force controller designed for rigid-body systems but acting on a manipulation system with a deformable object. The planar manipulation system is the two Dof's depicted in fig. 2-a). It has the contact points in ${ }^{2}(0,2)$ and $(2,2)$, the prismatic joint in $(2,1)$, and stiffness, damping and inertia matrices normalized to the identity matrix. The Jacobian and the grasp matrix take the following values

$$
\mathbf{J}=\left[\begin{array}{cc}
-2 & 0 \\
0 & 0 \\
-2 & -1 \\
2 & 0
\end{array}\right] ; \mathbf{G}=\left[\begin{array}{cccc}
1 & 0 & 1 & 0 \\
0 & 1 & 0 & 1 \\
0 & -1 & 0 & 1
\end{array}\right]
$$

Observe that the system is defective, cf. [11], $\operatorname{ker}\left(\mathbf{J}^{T}\right) \neq$ $\emptyset$. The controlled output is $\mathbf{e}_{t i}(2)$ : the projection of the force vector $\mathbf{t}$ onto the null space of $\mathbf{G}$, i.e. the subspace of reachable internal forces which, in the case study, is $\mathcal{R}_{t i, \tau}=\operatorname{im}\left(\left[\begin{array}{llll}1 & 0 & -1 & 0\end{array}\right]\right)$

The force control block diagram is reported in fig. 3 . It consists of two loops: the internal one is the stabilizing (cf. [10]) PD controller of joint positions while the external one is the force loop composed by the internal model and the Jacobian transpose.

The internal model principle guarantees that when the input is a unit step the magnitude of the output $\mathbf{e}_{t i}$ reaches the unit value.

Unfortunately the controller action moves the grasped object along the rigid body coordinate subspace, $\operatorname{im}\left(\Gamma_{u c}\right)=\operatorname{im}\left[\begin{array}{lll}-0.76 & 0.38 & 0.38\end{array}\right]$. Simulation results are reported in fig. 4 and 5 . The final config-

\footnotetext{
${ }^{2}$ With respect to the depicted reference frame.
} 


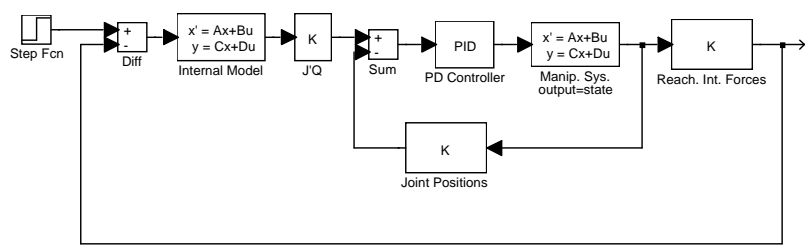

Figure 3: Two-loops internal force control law.

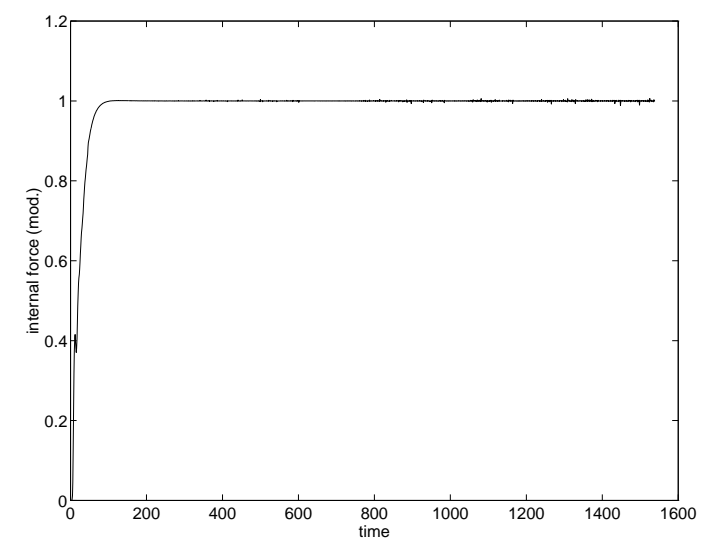

Figure 4: Simulation result of block diagram 3, reachable nternal force.

uration of the manipulation system looks like the one depicted in fig. 2-b). Obviously this is a dangerous maneuver in applications like those pictorially described in fig. 1. The paper contribution consists in proposing a feedback internal force controller whose action results to be decoupled from the rigid-body object dynamics.

\section{Object-motion decoupling control of internal forces}

This section is aimed at the analysis of the objectmotion decoupling control of internal forces for general grasping mechanisms.

Definition 1 Consider the dynamic system (1). A control law of the internal forces $\mathbf{e}_{t i}$ is decoupled with respect to the coordinate rigid-body motions $\mathbf{e}_{u c}$, if there exists a linear combination $\mathbf{B}_{\tau} \mathbf{U}$ of the input-matrix

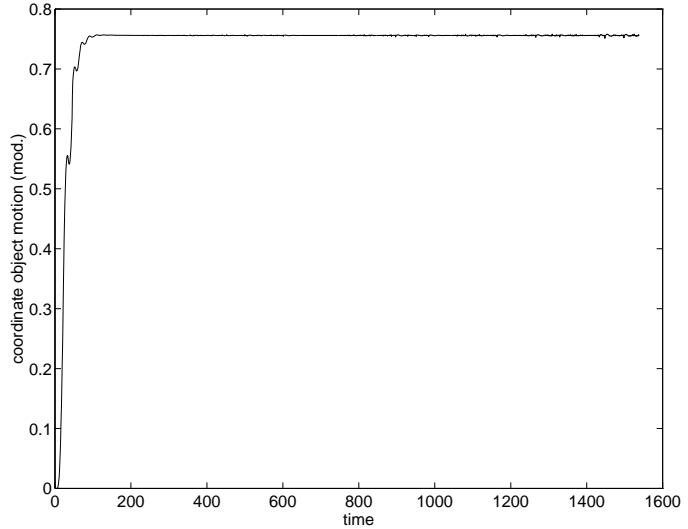

Figure 5: Simulation result of block diagram 3, coordinate rigid-body object positions.

columns such that, for zero initial condition, the input $\tau=\mathbf{U} \tau_{n}$ only affects the internal forces $\mathbf{e}_{t i}$ while $\mathbf{e}_{u c}$ remains identically zero.

The following theorem shows how this definition can be considered a structural property of general (nonindeterminate) manipulation systems.

Assume that:

H1: The manipulation mechanism is not indeterminate, i.e. $\operatorname{ker}\left(\mathbf{G}^{T}\right)=\mathbf{0}$;

H2: $\operatorname{ker}\left(\Gamma_{u c}^{T}\right)$ is $\mathbf{M}_{o}^{-1} \mathbf{G K G}^{T}$-invariant Then the following theorem holds.

Theorem 1 Consider the linearized manipulation system (1). Under the hypothesis $\mathbf{H 1 - H 2}$, there exists a stabilizing state feedback $\mathbf{F}$ and a matrix $\mathbf{U}$ such that

- the decoupling condition of Definition 1 holds

- $\operatorname{im}\left(\mathbf{E}_{t i}<\mathbf{A}+\mathbf{B}_{\tau} \mathbf{F} \mid \mathbf{B}_{\tau}>\right)=\operatorname{im}\left(\mathbf{E}_{t i}\right)$.

being $<\mathbf{A} \mid \mathbf{B}>=\left[\begin{array}{llll}\mathbf{B} \mathbf{A B} \mathbf{A}^{2} \mathbf{B} & \cdots & \mathbf{A}^{n-1} \mathbf{B}\end{array}\right]$ where $\mathbf{A} \in \mathbb{R}^{n \times n}$.

Note that hypothesis $\mathbf{H 2}$ is technically in nature and has been introduced in order to simplify the proof of the theorem.

Theorem 1 states both the decoupling property and the complete output reachability of internal forces $\mathcal{R}_{t i, \tau}$ (3). If the sensor system of the robotic manipulator is not able to measure the object position and velocity, a state observer is needed in the control loop. This commonly occurs when robots are equipped with tactile sensors. If contact force and joint position sensors are chosen, the local state detectability from the measured outputs has been proved, cf. [10].

The proof of the decoupling theorem is reported in [9]. It has been developed in a framework proper of the 
geometric approach to the multivariable control design, $[2,14]$.

\section{Algebraic output decoupling of inter- nal forces}

This section shows how the decoupling control of internal forces can be obtained by means of an algebraic output feedback control from the sensed output consisting of contact forces $\mathbf{t}$ and of manipulator joint positions $\mathbf{q}$ whose output relationship for the linearized model is

$$
\begin{aligned}
\mathbf{y}_{m} & =\mathbf{C}_{m} \mathbf{x} \\
\mathbf{C}_{m} & =\left[\begin{array}{cccc}
\mathbf{I}_{q \times q} & \mathbf{0} & \mathbf{0} & \mathbf{0} \\
\mathbf{K J} & -\mathbf{K G}^{T} & \mathbf{B J} & -\mathbf{B G}^{T}
\end{array}\right] .
\end{aligned}
$$

Under the assumptions $\mathbf{H 1}$ and from (4), the nullspace of $\mathbf{C}_{m}$ is easily computed as

$$
\operatorname{ker}\left(\mathbf{C}_{m}\right)=\left[\begin{array}{ccc}
\mathbf{0} & \mathbf{0} & \mathbf{0} \\
\mathbf{0} & \mathbf{I} & \mathbf{0} \\
\boldsymbol{\Gamma}_{q c} & \mathbf{0} & \operatorname{ker}(\mathbf{J}) \\
\boldsymbol{\Gamma}_{u c} & -\alpha \mathbf{I} & \mathbf{0}
\end{array}\right] .
$$

The following theorem, proven in [9], shows that the decoupling control of internal forces, stated in Definition 1, can be obtained through an algebraic feedback of the sensed output $\mathbf{y}_{m}$.

Theorem 2 Consider the linearized manipulation system (1). Under hypotheses $\boldsymbol{H 1 - H 2 , ~ t h e r e ~ e x i s t s ~ a n ~ a l - ~}$ gebraic output feedback $\mathbf{L}$ of $\mathbf{y}_{m}$ and a matrix $\mathbf{U}$ such that

- the decoupling condition of Definition 1 holds and

- $\operatorname{im}\left(\mathbf{E}_{t i}<\mathbf{A}+\mathbf{B}_{\tau} \mathbf{C}_{m} \mathbf{L} \mid \mathbf{B}_{\tau} \mathbf{U}>\right)=\operatorname{im}\left(\mathbf{E}_{t i}\right)$.

\section{Decoupled internal force control ex- ample}

The case study has been introduced in Section 5 together with the non-decoupling control law of fig. 3 . Simulation results of Section 5 are here compared with those obtained by using the decoupling controller of Theorem 1.

The state-feedback gain $\mathbf{F}$ and the input selection matrix $\mathbf{U}$ of Theorem 1are obtained as

$$
\mathbf{F}=\left[\begin{array}{cccccccccc}
5 & 2 & 4 & -4.5 & -4.5 & 4 & 2 & 4 & -4.5 & -4.5 \\
2 & -6 & 11 & 10 & 10 & 2 & -7 & 11 & 10 & 10
\end{array}\right]
$$

and

$$
\mathbf{U}=\left[\begin{array}{c}
-0.16 \\
0.99
\end{array}\right]
$$

The controlled output is $\mathbf{e}_{t i}$ : the projection of the force vector $\mathbf{t}$ onto the null space of $\mathbf{G}$, i.e. the reachable

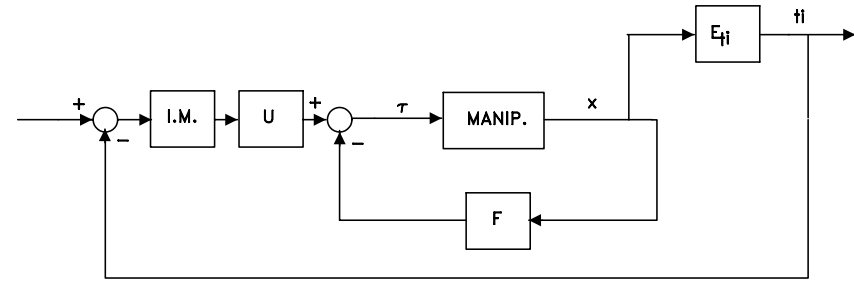

Figure 6: State-feedback control decoupling internal forces from object motions.

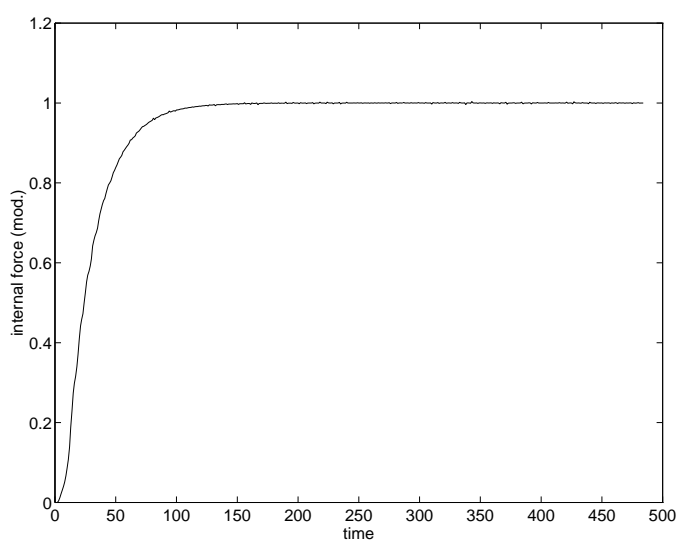

Figure 7: Simulation result of block diagram 6 , reachable internal force.

internal forces which, in the case study, are $\mathcal{R}_{t i, \tau}=$ $\operatorname{im}\left(\left[\begin{array}{llll}1 & 0 & -1 & 0\end{array}\right]\right)$.

Again the internal model principle guarantees that, with a unit step as input, the internal force $\mathbf{e}_{t i}$ reaches (in magnitude) the unit, but unlike the controller of fig. 6, the decoupling control of fig. 6 does not affect the rigid-body object motion which remains identically zero. In other words, the proposed internal force controller allows one to squeeze the object, see fig. 7 , in a way that the manipulated object does not change its position, see fig. 8 .

Fig.9 pictorially describes the squeezing action by the decoupling controller.

\section{Conclusions}

In this paper we considered the problem of controlling internal forces of grasps with general kinematics.

Special attention was devoted to manipulation systems with significant compliance at the contacts. As a consequence, the object dynamics was taken into account in controlling the internal forces.

After characterizing the rigid-body object motions 


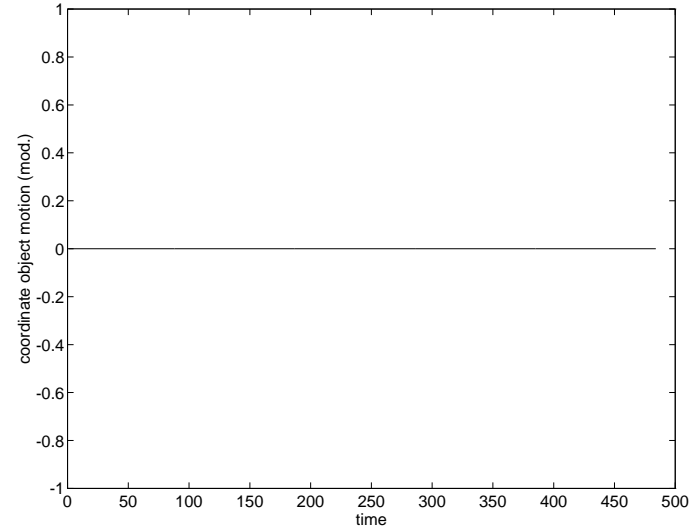

Figure 8: Simulation result of block diagram 6, coordinate rigid-body object positions.

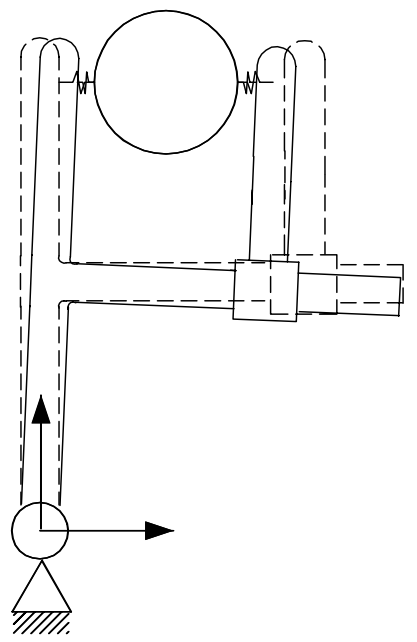

Figure 9: Decoupling internal force control.

and the reachable contact forces, we focused on the problem of synthesizing an internal force control law which does not interact with the rigid-body object motion.

The geometric approach is used throughout the paper whose main result states that there always exists a feedback control law that decouples the internal force control action from the object dynamics.

Notice that such a decoupling property can be considered as a structural property of manipulation systems with general kinematics.

\section{References}

[1] P.N. Akella, and M.R. Cutkosky, "Contact transition control with semiactive soft fingertips," in
IEEE Trans. Robotics Automat., Vol. 11, No. 6, pp. 859-867, 1995.

[2] Basile G. and Marro G. Controlled and conditioned invariants in linear system theory, New Jersey, Prentice Hall, 1992.

[3] A. Bicchi, "Force distribution in multiple wholelimb manipulation," in Proc. IEEE Int. Conf. Robotics Automat., pp. 196-201, 1993.

[4] A. Bicchi, C. Melchiorri and D. Balluchi, "On the mobility and manipulability of general multiple limb robots," IEEE Trans. on Robotics and Automat., vol. 11, n. 2, 1995.

[5] A. Bicchi, G. Canepa, D. De Rossi, P. Iacconi, E.P. Scilingo, "A sensorized minimmally invasive surgery tool for detecting tissutal elastic properties," in Proc. IEEE Int. Conf. Robotics Automat., vol.1, pp. 884-888, 1996.

[6] S. Ku, S.E. Salcudean , "Design and control of a teleoperated microgripper for microsurgery," in Proc. IEEE Int. Conf. Robotics Automat., vol.1, 1996.

[7] M.T. Mason and J.K. Salisbury, Robot hands and the mechanics of manipulation, The MIT Press, Cambridge MA, 1985.

[8] L. Meirovitch, Analytical methods in vibrations, Macmillan Pub. Co., Inc. New York. 1967.

[9] D. Prattichizzo P. Mercorelli and F. Barbagli, "Dynamic Control of Internal Force for Visco-Elastic Contact Grasps," Internal Report N. 123995, Faculty of Engineering, University of Siena.

[10] D. Prattichizzo and A. Bicchi, "Dynamic analysis of mobility and graspability of general manipulation systems", IEEE Transactions on Robotics and Automation, Vol. 14, No. 2, April 1998.

[11] D. Prattichizzo, and A. Bicchi, "Consistent task specification for manipulation systems with general kinematics," ASME Transactions Journal on Dynamics, System, Measurements and Control, Dec. 1997.

[12] R.H. Taylor et. al., "Taming the Bull: safety in a precise surgical robot," in Proc. IEEE Int. Conf. Robotics Automat., vol. 1, 1991.

[13] J.C. Trinkle, "Dexterous manipulation planning and execution of an enveloped slippery workpiece," in Proc. IEEE Int. Conf. Robotics Automat., 1993.

[14] W.M. Wonham, Linear multivariable control: a geometric approach, New York, Springer-Verlag, 1979. 